Please cite this paper as follows:

H.-K. Hong and R. Vaicaitis, Nonlinear Response of Double Wall Sandwich Panels, Journal of Structural Mechanics, Vol.12, No.4, pp.483-503, 1984. 
This article was downloaded by: [National Taiwan University]

On: 15 September 2011, At: 17:46

Publisher: Taylor \& Francis

Informa Ltd Registered in England and Wales Registered Number: 1072954 Registered office: Mortimer House, 37-41 Mortimer Street, London W1T 3J H, UK

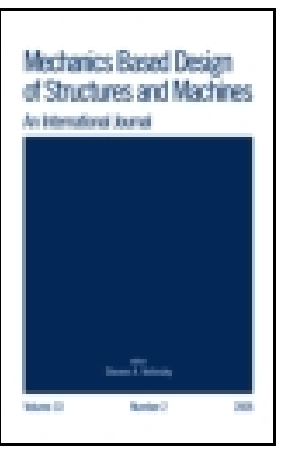

\section{J ournal of Structural Mechanics}

Publication details, including instructions for authors and subscription information: http:// www. tandfonline.com/loi//mbd18

\section{Nonlinear Response of Double Wall Sandwich Panels**}

H. - K. Hong ${ }^{\text {a }} \&$ R. Vaicaitis ${ }^{a}$

${ }^{a}$ COLUMBIA UNIVERSITY, NEW YORK, NEW YORK

Available online: 25 J un 2007

To cite this article: H.-K. Hong \& R. Vaicaitis (1984): Nonlinear Response of Double Wall Sandwich Panels**, J ournal of Structural Mechanics, 12:4, 483-503

To link to this article: http:// dx. doi.org/ 10.1080/03601218408907483

\section{PLEASE SCROLL DOWN FOR ARTICLE}

Full terms and conditions of use: http://www.tandfonline.com/page/terms-and-conditions

This article may be used for research, teaching and private study purposes. Any substantial or systematic reproduction, re-distribution, re-selling, loan, sub-licensing, systematic supply or distribution in any form to anyone is expressly forbidden.

The publisher does not give any warranty express or implied or make any representation that the contents will be complete or accurate or up to date. The accuracy of any instructions, formulae and drug doses should be independently verified with primary sources. The publisher shall not be liable for any loss, actions, claims, proceedings, demand or costs or damages whatsoever or howsoever caused arising directly or indirectly in connection with or arising out of the use of this material. 
J. STRUCT. MECH., 12(4), 483-503 (1984-85)

\title{
Nonlinear Response of Double Wall Sandwich Panels*
}

\author{
H.-K. Hong ${ }^{\dagger}$ and R. Vaicaitis \\ Columbia University \\ NeW York, New YoRK
}

\begin{abstract}
An analytical study is presented to predict the nonlinear response of a double wall sandwich panel system that is subjected to random-type loading. Viscoelastic and nonlinear spring-dashpot models are chosen to characterize the behavior of the core. The nonlinear panel response is obtained by utilizing modal analyses and Monte Carlo simulation techniques. Numerical results include the response spectral densities, root-mean-square responses, and probability density function histograms. 1t is found that by proper selection of the dynamic parameters and damping characteristics, the structural response can be significantly reduced.
\end{abstract}

\section{INTRODUCTION}

Information available in the literature and from ongoing research on the response of surface panels of various transportation vehicles that are ex-

*Communicated by J. T. P. Yao.

tPresently with the Department of Civil Engineering, National Taiwan University, Taipei, Taiwan.

483

Copyright $(9) 1985$ by Marcel Dekker, lnc.

$0360-1218 / 84 / 1204-0483 \$ 3.50 / 0$ 
posed to high intensity inputs indicates a need to reduce these response levels to acceptable limits [1,2]. Past studies have demonstrated that sandwich panels can achieve a significant amount of response reduction [3-7]. However, these studies were limited to linear models. Under high intensity inputs, soft core materials, and/or thin face plate constructions, linear theory becomes invalid. Therefore, an analytical study of nonlinear response of a double wall sandwich-type panel system is undertaken in this paper.

The governing differential equations for nonlinear vibration of a double wall construction shown in Fig. 1 are developed for the case in which the core material is taken to be very soft, so that bending and shearing stresses can be neglected and, consequently, the core can be described by a uniaxial constitutive law. Linear viscoelastic and nonlinear spring-dashpot models are chosen to characterize behavior of the core. Both face plates are modeled according to thin plate, large deflection theory $[8,9]$. The input acting on the external (top) face plate is assumed to be a random pressure field.

Several methods have been proposed to study random vibration of nonlinear systems [10-12]. Among the most widely used are the Fokker-Planck equation solutions $[10,11,13]$, perturbation method [14, 15], stochastic linearization [16, 17], and the Monte Carlo simulation [18, 24]. Exact solutions of the Fokker-Planck equation are available only for a few simple cases. The perturbation method is usually limited to one or two degree of freedom systems and is valid only for weakly nonlinear cases. Stochastic linearization, although used for problems with strong nonlinearities [18, 19], may not yield meaningful results for the complex nonlinear problem considered in this study. To circumvent these difficulties, a Monte Carlo approach is employed to study the nonlinear response of a double wall panel system. In this case the time histories of the random input processes are simulated $[20,24]$ and the solution of the governing equations is obtained in a time domain. A modal analysis is uitilized to decompose the vibrations of the face plates and the coupled system is solved by a Galerkin-like procedure [9]. For verification, a comparison is made between results of the simulation and power spectral density approaches for special linearized cases.

The objective of the present study is to develop an analytical model that is capable of predicting nonlinear response of the double wall sandwich construction and to demonstrate that such a model could be used to minimize response of the bottom plate. By proper selection of the dynamic parameters and damping characteristics, the response of the bottom plate can be reduced, even though the vibration levels of the top plate are large. Such a model could be used to study the response of compliant walls to convective random inputs and to minimize noise transmission into aircraft and other transportation vehicles. 


\section{RANDOM RESPONSE OF A DOUBLE WALL SYSTEM}

The double wall system is composed of a soft core and two elastic face plates, as shown in Fig. 1. To assess the dynamic response characteristics of the system, various structural models that include geometric and damping nonlinearities and the viscoelastic properties of the core are considered in the formulation.

For vibration of the elastic face plates, both classical linear and the von Karman nonlinear theories are considered $[8,9,25]$. Several linear and nonlinear models are constructed to represent the behavior of the core material and to investigate its effect on the overall response of the system. In the linear case the response analysis is performed in a frequency domain, utilizing a power spectral density approach [3]. The nonlinear response is calculated in a time domain by a Monte Carlo method [20]. The analysis presented herein is limited to a thick core of dimension 0.25 to 2 in. The details and numerical results of a linear formulation can be found in Ref, 3 .

The geometry of the double wall sandwich panel system used for nonlinear deflection response calculations is shown in Fig. 1. The top and bottom plates are simply supported on all four edges. Following von Karman plate theory, the plate motions are modeled by four nonlinear partial differential equations for the vertical deflections $w_{7}$ and $w_{B}$ and the Airy stress functions $F_{T}$ and $F_{B}[8,9,25-27]$,

$$
\begin{aligned}
m_{T} \ddot{w}_{T}+c_{T} \dot{w}_{T}+ & D_{T} \nabla^{4} w_{T}-\frac{\partial^{2} F_{T}}{\partial y^{2}} \frac{\partial^{2} w_{T}}{\partial x^{2}}-\frac{\partial^{2} F_{T}}{\partial x^{2}} \frac{\partial^{2} w_{T}}{\partial y^{2}}+2 \frac{\partial^{2} F_{T}}{\partial x \partial y} \frac{\partial^{2} w_{T}}{\partial x \partial y} \\
& +m_{S} \ddot{w}_{T} / 3+m_{S} \ddot{w}_{B} / 6+K_{S}\left[w_{T}-w_{B}\right]+p_{E}(x, y, t)=0
\end{aligned}
$$

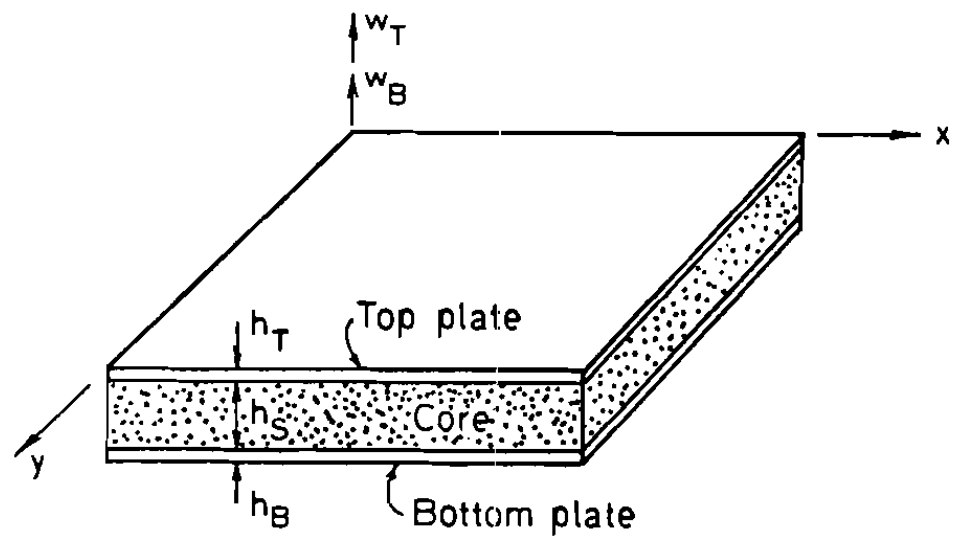

Fig. 1 Geometry of double wall sandwich panel. 


$$
\begin{gathered}
\frac{\nabla^{4} F_{T}}{E_{T} h_{T}}=\left(\frac{\partial^{2} w_{T}}{\partial x \partial y}\right)^{2}-\frac{\partial^{2} w_{T}}{\partial x^{2}} \frac{\partial^{2} w_{T}}{\partial y^{2}} \\
m_{B} \ddot{w}_{B}+c_{B} \dot{w}_{B}+D_{B} \nabla^{4} w_{B}-\frac{\partial^{2} F_{B}}{\partial y^{2}} \frac{\partial^{2} w_{B}}{\partial x^{2}}-\frac{\partial^{2} F_{B}}{\partial x^{2}} \frac{\partial^{2} w_{B}}{\partial y^{2}}+2 \frac{\partial^{2} F_{B}}{\partial x \partial y} \frac{\partial^{2} w_{B}}{\partial x \partial y} \\
+m_{S} \ddot{w}_{B} / 3+m_{S} \ddot{w}_{T} / 6+K_{S}\left[w_{B}-w_{T}\right]-p(x, y, 0, t)=0 \\
\frac{\nabla^{4} F_{B}}{E_{B} h_{B}}=\left(\frac{\partial^{2} w_{B}}{\partial x \partial y}\right)^{2}-\frac{\partial^{2} w_{B}}{\partial x^{2}} \frac{\partial^{2} w_{B}}{\partial y^{2}}
\end{gathered}
$$

where $m, c, D, E$, and $h$ are the mass, damping coefficient, plate stiffness, modulus of elasticity, and plate thickness, respectively. Subscripts $T, B$, and $S$ denote top and bottom plates and the core, respectively. The $K_{S}$ is either a linear or nonlinear operator that describes the behavior of the soft core, $\nabla^{4}$ is the biharmonic operator $\nabla^{4}=\partial^{4} / \partial x^{4}+2 \partial^{4} / \partial x^{2} \partial y^{2}+\partial^{4} / \partial y^{4}$, and $p(x, y$, $0, t)$ and $p_{E}(x, y, t)$ are the acoustic and input pressures that act on the bottom and top plates. Equations 1 and 3 express Newton's second law in the vertical direction, while Eqs. 2 and 4 ensure the compatibility of inplane strains. Defining the Airy stress functions for both plates, in terms of the membrane in-plane forces, $N_{x}, N_{y}$, and $N_{x y}$,

$$
\left.\begin{array}{l}
N_{x}=\partial^{2} F / \partial y^{2} \\
N_{y}=\partial^{2} F / \partial x^{2} \\
N_{x y}=N_{y x}=-\partial^{2} F / \partial x \partial y
\end{array}\right\}
$$

the equilibrium requirements are satisfied. Subscripts $T$ and $B$ have been dropped, since for each $F$ there is one set of equations, Eq. 5 .

The boundary conditions corresponding to vertical deflections $w_{T}$ and $w_{B}$ are those of simply supported plates. Exact boundary conditions for the Airy stress function $F$ are very complicated and, for the present study, inplane boundary conditions are satisfied on the average [25]. Thus,

$$
\left.\begin{array}{l}
\int_{0}^{L_{y}} \int_{0}^{L_{x}} \frac{\partial u}{\partial x} d x d y=0 \\
\int_{0}^{L_{x}} \int_{0}^{L_{y}} \frac{\partial v}{\partial y} d y d x=0 \\
\left.\bar{N}_{x y}\right|_{x=0, L_{x}}=0 \\
\left.\bar{N}_{y x}\right|_{y=0, L_{y}}=0
\end{array}\right\}
$$

where 


$$
\left.\begin{array}{l}
\bar{N}_{x y} \equiv \frac{1}{L_{y}} \int_{0}^{L_{y}} N_{x y} d y \\
\bar{N}_{y x} \equiv \frac{1}{L_{x}} \int_{0}^{L_{x}} N_{y x} d x
\end{array}\right\}
$$

The terms $u$ and $v$ are in-plane displacements, which can be expressed in terms of the Airy stress function and the vertical deflection,

$$
\left.\begin{array}{l}
\frac{\partial u}{\partial x}=\frac{1}{E h}\left(\frac{\partial^{2} F}{\partial y^{2}}-v \frac{\partial^{2} F}{\partial x^{2}}\right)-\frac{1}{2}\left(\frac{\partial w}{\partial x}\right)^{2} \\
\frac{\partial v}{\partial y}=\frac{1}{E h}\left(\frac{\partial^{2} F}{\partial x^{2}}-v \frac{\partial^{2} F}{\partial y^{2}}\right)-\frac{1}{2}\left(\frac{\partial w}{\partial y}\right)^{2}
\end{array}\right\}
$$

in which $v$ is Poisson's ratio. The first two conditions given in Eq. 6 imply no in-plane stretching of the plane midsurface, while the last two specify vanishing in-plane shear at the plate boundaries.

To solve Eqs. 1-4, vertical deflections of the top and bottom plates are expressed in terms of the simply supported plate modes:

$$
\begin{aligned}
& w_{T}(x, y, t)=\sum_{m=1}^{\infty} \sum_{n=1}^{\infty} A_{m n}^{T}(t) X_{m n}(x, y) \\
& w_{B}(x, y, t)=\sum_{m=1}^{\infty} \sum_{n=1}^{\infty} A_{m n}^{B}(t) X_{m n}(x, y)
\end{aligned}
$$

where $A_{m n}^{T}$ and $A_{m n}^{B}$ are generalized coordinates of the top and bottom plates, respectively, and $X_{m n}=\sin \left(m \pi x / L_{x}\right) \sin \left(n \pi y / L_{y}\right)$.

Substitution of Eqs. 9 and 10 into Eqs. 2 and 4 yields two inhomogeneous partial differential equations in $F$. The solution for $F$ may then be written as

$$
F=F_{p}+F_{h}
$$

where $F_{p}$ and $F_{h}$ denote the particular and homogeneous solutions, respectively. Since both equations for $F_{T}$ and $F_{B}$ are of the same form, only one equation is given and the subscripts and superscripts are dropped for brevity. A particular solution for Airy stress function can be obtained, following the procedures given in Refs. 25-28, as

$$
\begin{aligned}
F_{p}= & \frac{E h}{4}\left(\frac{L_{x}}{L_{y}}\right)^{2} \sum_{m}^{(n r} \sum_{n} \sum_{r}^{m s} \sum_{s}^{0)} A_{m n} A_{r s} m s(n r-m s) \\
& \times\left\{\left[(m+r)^{2}+\left(\frac{L_{x}}{L_{y}}\right)^{2}(n+s)^{2}\right]^{-2} \cos \frac{(m+r) \pi x}{L_{x}} \cos \frac{(n+s) \pi y}{L_{y}}\right.
\end{aligned}
$$




$$
\begin{aligned}
& \left.+\left[(m-r)^{2}+\left(\frac{L_{x}}{L_{y}}\right)^{2}(n-s)^{2}\right]^{-2} \cos \frac{(m-r) \pi x}{L_{x}} \cos \frac{(n-s) \pi y}{L_{y}}\right\} \\
& +\frac{E h}{4}\left(\frac{L_{x}}{L_{y}}\right)^{2} \sum_{m} \sum_{n} \sum_{r} \sum_{s} A_{m n} A_{r s} m s(n r+m s) \\
& \times\left\{\left[(m+r)^{2}+\left(\frac{L_{x}}{L_{y}}\right)^{2}(n-s)^{2}\right]^{-2} \cos \frac{(m+r) \pi x}{L_{x}} \cos \frac{(n-s) \pi y}{L_{y}}\right. \\
& \left.+\left[(m-r)^{2}+\left(\frac{L_{x}}{L_{y}}\right)^{2}(n+s)^{2}\right]^{-2} \cos \frac{(m-r) \pi x}{L_{x}} \cos \frac{(n+s) \pi y}{L_{y}}\right\}
\end{aligned}
$$

For the case of in-plane boundary conditions that are satisfied on the average, the homogeneous solution can be written as

$$
F_{h}=C_{7} x^{2} / 2+C_{8} y^{2} / 2-C_{9} x y
$$

where $C_{7}, C_{8}$, and $C_{9}$ are constants of integration that are to be determined from the boundary conditions specified in Eq. 6. Combining Eqs. 5, 11, and 13 ,

$$
\left.\begin{array}{l}
N_{x}=\partial^{2} F_{p} / \partial y^{2}+C_{8} \\
N_{y}=\partial^{2} F_{p} / \partial x^{2}+C_{7} \\
N_{x y}=-\partial^{2} F_{p} / \partial x \partial y+C_{9}
\end{array}\right\}
$$

Using Eqs. 6 and 8, together with the expression for the Airy stress function $F$ and the solution for the plate deflection $w$, the constants of integration reduce to

$$
\begin{aligned}
& C_{7}=\frac{\pi^{2} E h}{8\left(1-v^{2}\right)} \sum_{m=1}^{\infty} \sum_{n=1}^{\infty} A_{m n}^{2}\left(v \frac{m^{2}}{L_{x}^{2}}+\frac{n^{2}}{L_{y}^{2}}\right) \\
& C_{8}=\frac{\pi^{2} E h}{8\left(1-v^{2}\right)} \sum_{m=1}^{\infty} \sum_{n=1}^{\infty} A_{m n}^{2}\left(\frac{m^{2}}{L_{x}^{2}}+v \frac{n^{2}}{L_{y}^{2}}\right) \\
& C_{9}=0
\end{aligned}
$$

which can then be substituted into Eq. 13 to determine the homogeneous solution for the Airy stress function.

Having determined $F$, Eqs. 1 and 3 are satisfied in a Galerkin sense. The resulting system of nonlinear differential equations for the top plate are 


$$
\begin{aligned}
& m_{T} \ddot{A}_{l j}^{T}+c_{T} \dot{A}_{i j}^{T}+\left[\left(i \pi / L_{x}\right)^{2}+\left(j \pi / L_{y}\right)^{2}\right]^{2} D_{T} A_{i j}^{T} \\
& \quad+A_{i j}^{T}\left[\left(i / L_{x}\right)^{2} \sum_{k} \sum_{l}\left(A_{k l}^{T}\right)^{2}\left(k^{2} / L_{i c}^{2}+v_{T} l^{2} / L_{y}^{2}\right)\right. \\
& \left.\quad+\left(j / L_{y}\right)^{2} \sum_{k} \sum_{l}\left(A_{k l}^{T}\right)^{2}\left(v_{T} k^{2} / L_{x}^{2}+l^{2} / L_{y}^{2}\right)\right] E_{T} h_{T} \pi^{4} /\left[8\left(1-v_{T}^{2}\right)\right] \\
& \quad+E_{T} h_{T}\left[\frac{\pi}{2 L_{y}}\right]^{4} \sum_{k} \sum_{i} \sum_{m} \sum_{n} \sum_{r} \sum_{i} A_{k l}^{T} A_{m n}^{T} A_{r s}^{T} Z_{l j k l m n r s} \\
& \quad+m_{S} \ddot{A}_{l k i}^{T / 3}+m_{S} \ddot{A}_{i j}^{B} / 6+K_{S}\left[A_{i j}^{T}-A_{l j}^{B}\right]=Q_{i j}^{T}(t)
\end{aligned}
$$

where the generalized random forces are

$$
Q_{i j}^{T}(t)=\frac{-4}{L_{x} L_{y}} \int_{0}^{L_{x}} \int_{0}^{L_{y}} p_{E}(x, y, t) \sin \left(i \pi x / L_{x}\right) \sin \left(j \pi y / L_{y}\right) d x d y
$$

The nonlinear stiffness coefficients $Z_{i j k \text { Imnrs }}$ in Eq. 16 are given in the Appendix. The resulting equations for the bottom plate are similar to those of Eqs. 16 and 17, except with the subscripts (and superscripts) $T$ and $B$ interchanged and the external random pressure $-p_{E}(x, y, t)$ replaced by the cavity backup pressure $p(x, y, 0, t)$. Then, the resulting system of equations for the generalize coordinates $A_{m n}^{T}$ and $A_{m n}^{B}$ can be solved numerically.

Due to the anticipated large deflections of the top plate and foamlike materials to be used in the core to isolate the motions of the double wall structural system, nonlinear and visccelastic models are constructed to represent the stiffness and damping characteristics of the core. Consider the case of a core material that is modeled as a combination of nonlinear springs and nonlinear dampers. The term $K_{S}\left[w_{T}-w_{B}\right]$ in Eqs. 1 and 3 can be written as

$$
\begin{aligned}
K_{S}\left[w_{T}-w_{B}\right]= & k_{1}\left(w_{T}-w_{B}\right)+k_{2}\left(w_{T}-w_{B}\right)^{2}+k_{3}\left(w_{T}-w_{B}\right)^{3} \\
& +c_{1}\left(\dot{w}_{T}-\dot{w}_{B}\right)+c_{2}\left(\dot{w}_{T}-\dot{w}_{B}\right)^{2}+c_{3}\left(\dot{w}_{T}-\dot{w}_{B}\right)^{3}
\end{aligned}
$$

where $k_{1}, k_{2}, k_{3}$ and $c_{1}, c_{2}, c_{3}$ are the stiffness and damping coefficients, respectively. The hard spring behavior is simulated by a positive cubic coefficient $k_{3}$. In this case, when the relative core displacement $\left(w_{T}-w_{B}\right)$ exceeds $\left(k_{1} / k_{2}\right)^{1 / 2}$, a state of strong nonlinearity is present. When the coefficient $k_{3}$ is negative, the effect of nonlinearity is that of a soft spring. The spring will buckle if the relative displacement exceeds the value of $\left(k_{1}\right)$ $\left.\left(-3 k_{3}\right)\right)^{1 / 2}$ [28]. By including the quadratic terms in Eq. 18, a system with asymmetric nonlinearity can be studied. It should be noted that a symmetric nonlinearity at the equilibrium position might be forced into asymmetric behavior if the system is excited about some mean position. 
When the core material exhibits linear viscoelastic behavior, the term $K_{S}\left[w_{T}-w_{B}\right]$ can be expressed as

$$
K_{S}\left[w_{T}^{\prime}-w_{B}\right]=\frac{1}{h_{S}} \int_{-\infty}^{\imath} E_{S}(t, \tau)\left\{\dot{w}_{T}(x, y, \tau)-\dot{w}_{B}(x, y, \tau)\right\} d \tau
$$

where $E_{S}(t, \tau)$ is the relaxation function of the viscoelastic material. For a nonaging viscoelastic material, $E_{S}(t, \tau)$ reduces to $E_{S}(t-\tau)$ and the expression on the right-hand side of Eq. 19 reduces to a convolution type integral. Thus,

$$
K_{S}\left[A_{k l}^{T}-A_{k l}^{B}\right]=\frac{1}{h_{S}} \int_{-\infty}^{1} E_{S}(t-\tau)\left\{\dot{A}_{k l}^{T}(\tau)-\dot{A}_{k l}^{B}(\tau)\right\} d \tau
$$

The terms $A_{k l}^{T}(\tau)$ and $A_{k l}^{B}(\tau)$ for $\tau<t$ are known during the numerical integration process of the system of equations given in Eq. 16.

Before step-by-step numerical integration can be implemented for the coupled system of nonlinear differential equations given for the top plate in Eq. 16 and a similar expression for the bottom plate, the time histories of the generalized random forces $Q_{i j}^{T}(t)$ and the solution for the enclosure pressure $p(x, y, 0, t)$ are needed. The solution for pressure in the enclosure is given in Ref. 29. The random time histories of the generalized random forces are generated utilizing a simulation technique for multidimensional and multivariate random processes [23]. To reduce computation time, the simulation procedure is modified to accommodate the fast Fourier transform (FFT) procedure [19-24].

The nonlinear system of equations that govern, the motion of the double wall system are coupled through inertia, damping, and stiffness terms. It is convenient to write the system in the following symbolic form:

$$
\begin{aligned}
& a_{T} \ddot{A}_{i j}^{T}+b_{S} \ddot{A}_{i j}^{B}+R_{i j}^{T}=0 \\
& a_{B} \ddot{A}_{i j}^{B}+b_{S} \ddot{A}_{i j}^{T}+R_{i j}^{B}=0
\end{aligned}
$$

where $a_{T}=m_{T}+m_{S} / 3, a_{B}=m_{B}+m_{S} / 3, b_{S}=m_{S} / 6$, and $R_{i j}^{T}$ and $R_{i j}^{B}$ include damping, stiffness, and forcing terms. The system of the nonlinear equations, Eqs. 21 and 22, can now be solved by standard numerical integration routines. Once the generalized coordinates $A_{i j}^{T}(t)$ and $A_{i j}^{B}(t)$ are known, displacements of the top and bottom plates are calculated from Eqs. 9 and 10. The root-mean-square values and the spectral densities of the deflection response can then be estimated. The spectral densities are calculated utilizing the FFT algorithm. It should be noted that, for the present nonlinear system, the response is non-Gaussian. 


\section{RANDOM PRESSURE INPUT}

In this section a brief discussion is given on the external random input, its spectral representation, and the simulation of the input sample functions, which are then used for a time domain analysis approach. The external noise acting on a structural panel is described, in general, by a random pressure process $p_{E}(x, y, t)$. The experimental pressure-time histories, if available, could be used directly in a time domain analysis. Depending on the nature of the input pressure, some simplifications of the randomness can be made to facilitate the recording and processing of data.

To provide the random response analysis of structures with nonlinearities and/or nonstationary inputs, a time domain Monte Carlo-type analysis is needed. Simulation techniques for multidimensional and multivariate Gaussian random processes with prescribed power spectral densities or evolutionary spectra can be utilized to generate sample inputs $[18,21]$. These simulations are greatly expedited by incorporating the fast Fourier transform (FFT) technique $[19,20,23]$. In the present study, random input pressures are assumed to be Gaussian and are simulated according to the procedure given in Ref. 18.

\section{NUMERICAL RESULTS}

Numerical results presented herein correspond to the double wall sandwich panel system shown in Fig. 1. The following set of parameters are selected for the study: The dimensions of the double wall panel are $L_{x}=20$ in. and $L_{y}=10 \mathrm{in}$. The structural response is computed at the center of the plate; i.e., $x=10 \mathrm{in}$. and $y=5 \mathrm{in}$. The top and bottom face plates are assumed to be made of aluminum, with material densities $p_{T}=\rho_{B}=$ $0.000251 \mathrm{lb}_{\mathrm{f}}-\mathrm{s}^{2} / \mathrm{in}^{4}$, moduli of elasticity $E_{T}=E_{B}=10^{7} \mathrm{psi}$, and Poisson's ratios $v_{T}=v_{B}=0.3$. The structural modal damping ratios of each plate are calculated from

$$
\zeta_{m n}=\zeta_{11}\left(\omega_{11} / \omega_{m n}\right)
$$

where $\omega_{m n}$ are the uncoupled natural frequencies. The fundamental modal damping ratio is taken to be $\zeta_{11}=0.02$.

The input random pressure $p_{E}$ that acts on the top plate of the double wall panel system is taken to be that of truncated Gaussian white noise, for which 
the spectral densities are

$$
S_{P}^{E}= \begin{cases}8.41 \times 10^{-7}\left(\mathrm{psi}^{2} / \mathrm{Hz},\right. & 0<f<1024 \mathrm{~Hz} \\ 0, & \text { otherwise }\end{cases}
$$

Spatial distribution of the random pressure is assumed to be uniform over the exterior surface of th: top plate. The spectral densities given in Eq. 24 correspond to a $110-\mathrm{dB}$ s sund level, based on a $1-\mathrm{Hz}$ bandwidth. The overall input sound pressure lev:l is $130.1 \mathrm{~dB}$. All calculations are based on a frequency bandwidth of $\Delta f=1 \mathrm{~Hz}$. The effect of the acoustic pressure $p(x$, $y, 0, t)$ is not included in the calculations.

To demonstrate accuracy of the time domain solution, a direct comparison of results is made between the power spectral density (PSD) and the Monte Carlo (MC) approaches. In Fig. 2, root-mean-square (rms) deflection response at the center of the top plate is plotted versus the input rms pressure. As can be observed from these results, agreement between these two methods is very good when the response is linear. In obtaining these results, the following data were used: equal top and bottom plate thicknesses $h_{T}=$ $h_{B}=0.032 \mathrm{in}$., core material-air spring with density $\rho_{S}=1.147 \times 10^{-7}$ $\mathrm{Ib}_{\mathrm{f}}-\mathrm{s}^{2} / \mathrm{in}^{4}$, thickness $h_{s}=1 \mathrm{in}$., spring constant $k_{1}=19.48 \mathrm{lb} / \mathrm{in}^{3}$, and $k_{2}=k_{3}=c_{1}=c_{2}=c_{3}=0$. Included also in Fig. 2 are the static and dynamic nonlinear deflection response of the top plate. Input for the static

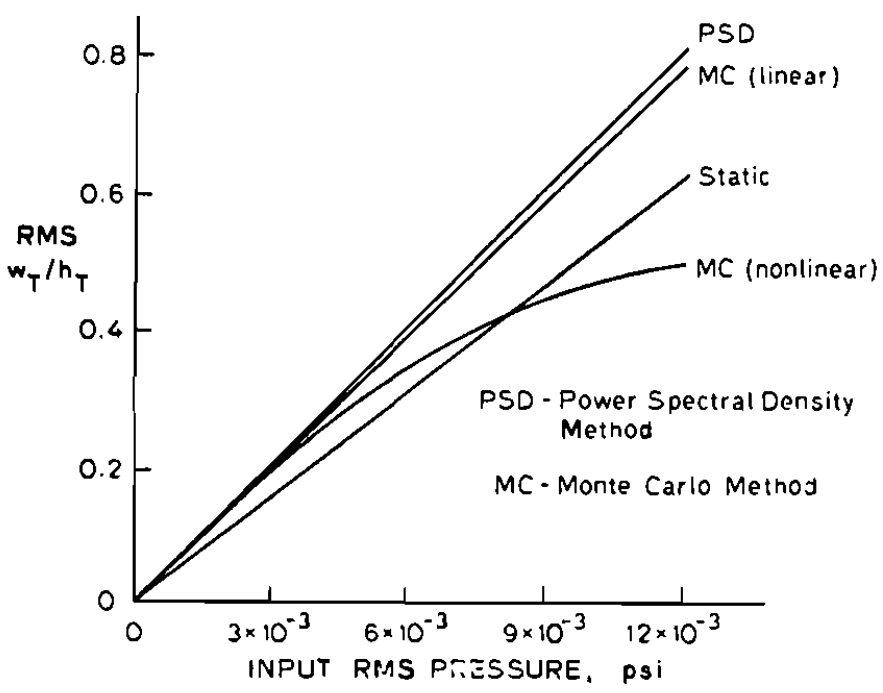

Fig. 2 Root-mean-square response of top plate. 


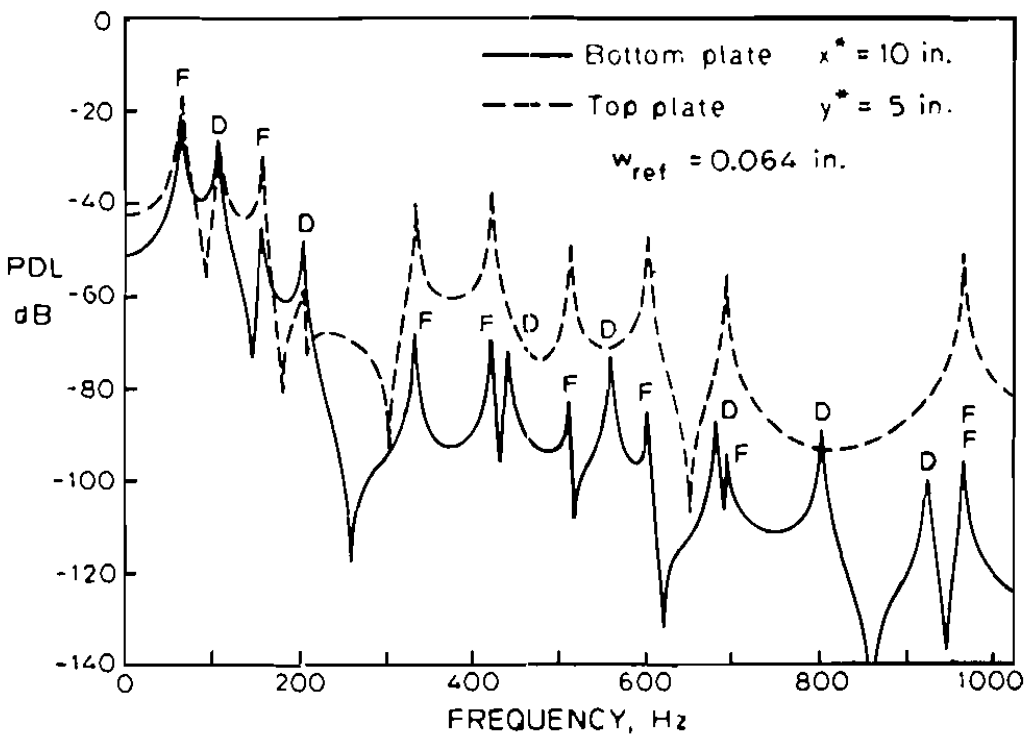

Fig. 3 Plate defizction levels ( $F=$ system flzxural mode, $D=$ system dilatational mode).

case is the rms value of the random pressures. Since the core, modeled by the air spring, is relatively stiff and the pressure inputs are relatively small, static response is linear and the loading is shared equally by the two plates. The deflection response spectral densities for the top and bottom plates, determined by the PSD method, are given in Fig. 3. The following data were used to obtain these results: $h_{T}=0.048$ in., $h_{B}=0.064$ in., $\rho_{S}=1.147 \mathrm{lb}_{\mathrm{r}}-\mathrm{s}^{2} / \mathrm{in}^{4}$, $h_{s}=1 \mathrm{in} ., k_{s}=2 \mathrm{lb} / \mathrm{in}^{3}$, and $g_{s}=0.03$. The abscissa is a logarithmic scale, called the plate deflection level (PDL), in units of decibels ( $\mathrm{dB})$,

$$
\operatorname{PDL}\left(x^{*}, y^{*}, \omega\right)=10 \log \left[S_{w}\left(x^{*}, y^{*}, \omega\right) \Delta \omega / w_{\text {ref }}^{2}\right]
$$

where $S_{w}$ is the deflection response spectral density, the reference deflection $w_{\text {ref }}$ is taken as $0.064 \mathrm{in}$., $x^{*}=10 \mathrm{in}$., and $y^{*}=5 \mathrm{in}$. The modes that are excited by the random pressure are indicated in Fig. 3 by the symbols $F$ and $D$, which refer to the flexural and dilatational modes, respectively, of the double wall system. As can be seen from Fig. 3, no distinct dilatational peaks are observed from the top plate at higher frequencies (above $300 \mathrm{~Hz}$ ). This is due to the fact that these dilatational. natural frequencies are very close to the uncoupled natural frequencies of the bottom plate and away from those of the top plate. Response levels at the higher frequencies are significantly lower for the bottom plate when compared to the response levels of the top plate. 
Shown in Fig. 4 are segments of the simulated time history of the input pressure and the response time histories of the top and bottom plates. Probability density functions of the panel deflections are plotted versus standard deviations in the form of a histogram in Fig. 5. For the purposes of comparison, Gaussian density functions are also included in the figure. From these results it can be observed that the nonlinear response of the top plate tends to exhibit non-Gaussian characteristics. The response of the bottom plate is linear and its amplitude distribution is very close to Gaussian.

A parametric study to determine the effects of variations in system parameters on the double wall panel response is performed. The effect of variations in the top plate thickness $h_{T}$ is illustrated in Fig. 6 where a Monte Carlo-type nonlinear analysis was performed to obtain these results. For the case $h_{T}=$ 0.064 in., response of the top plate is linear and distinct resonant peaks are observed in the response spectral density. However, when the response reaches the nonlinear range, resonant peaks above the fundamental mode are suppressed due to decoupling of the top and bottom plate modes. The flexural modes are most affected and the dilatational modes, except the
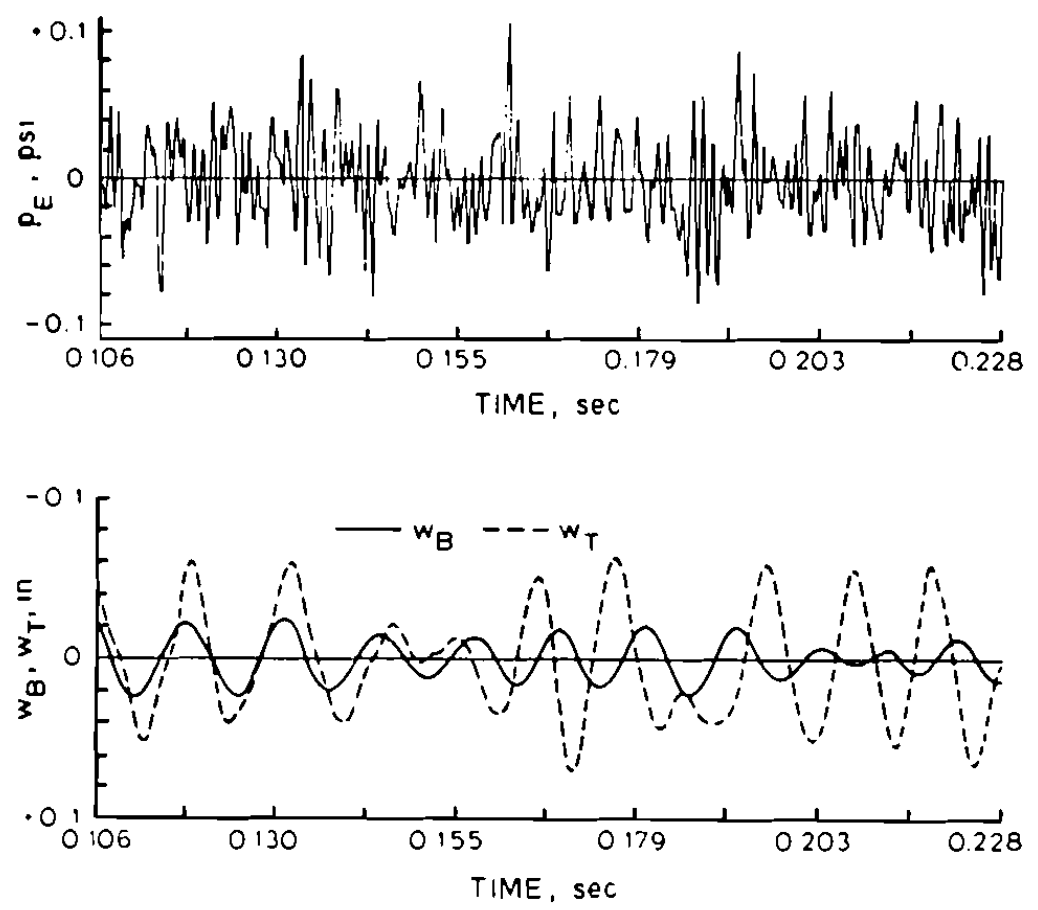

Fig. 4 Time histories of simulated input and plate responses. 


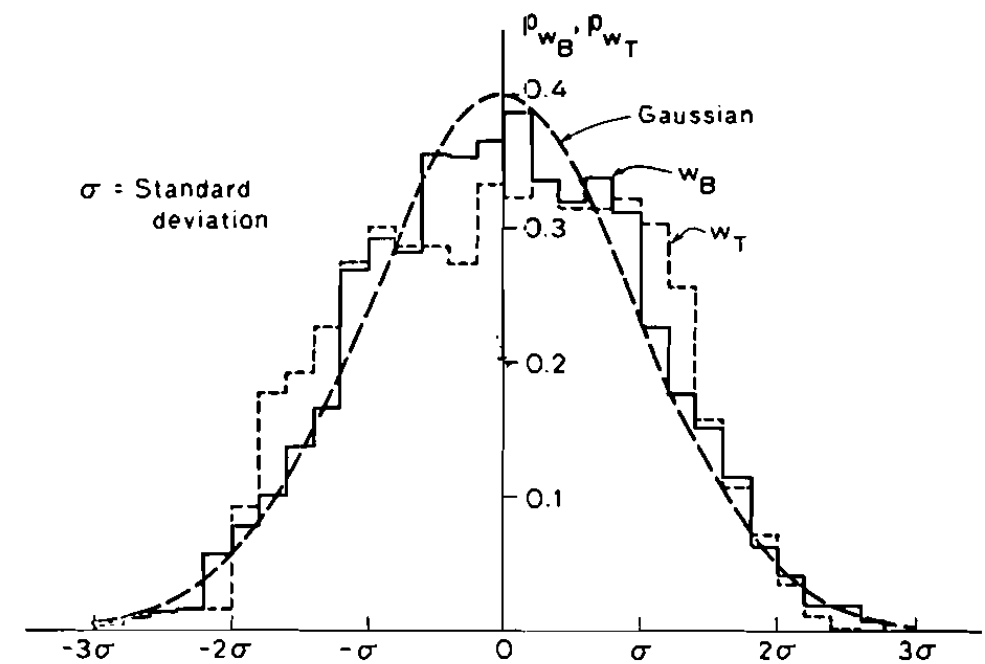

Fig. 5 Probability density function histogram of deflection response.

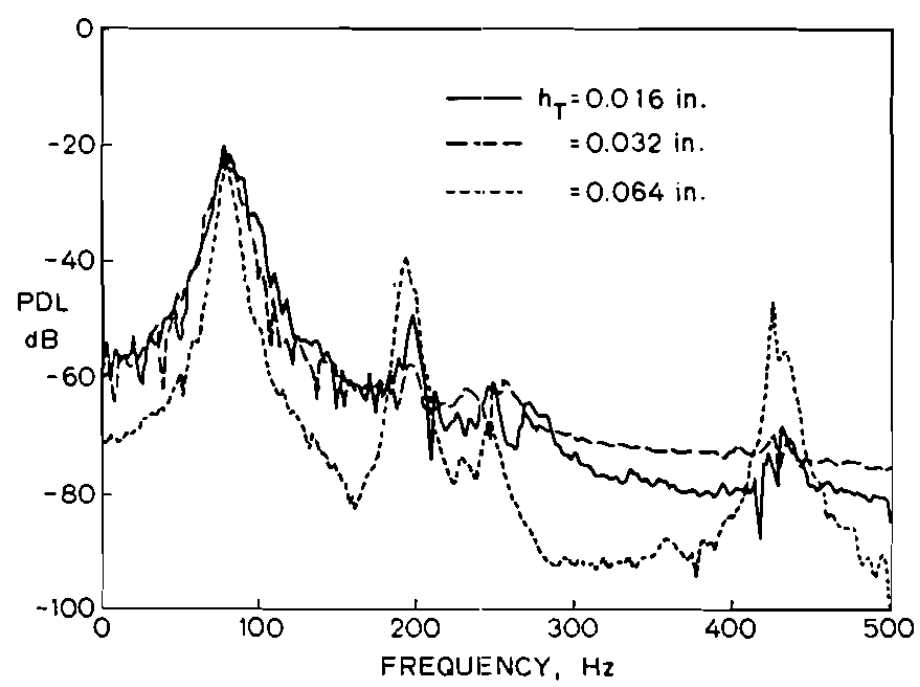

Fig. 6 Response of bottom plate for different top plate thicknesses.

fundamental ones, are excited to a lesser extent. Thus, significant reduction of the bottom plate response is achieved. However, response at the fundamental dilatational mode does not seern to be significantly affected by nonlinear response variations of the top plate. Since response is dominated by the fundamental mode, rms responses for the three cases considered are about 
the same. It should be noted that by decreasing the thickness of the top plate, surface density of the double wall sandwich construction is reduced. Thus, favorable gains in response reduction can be expected for a smaller amount of added weight through a design that consists of a thin top plate and a thicker bottom plate.

The effects of decreasing the stiffness of the core appear in Figs. 7 and 8. Results indicate that the response of the bottom plate is reduced over almost the entire range of frequencies and the resonant peaks are shifted to lower frequencies. A softer core material, therefore, can accommodate more deformation. As a result, à significant decrease in bottom plate response may be obtained by reducing the thickness of the top plate and decreasing the core stiffness. This could be attributed to large deflections of the top plate and the core, which contribute to most of the deformation of the coupled system and thus alleviate response of the bottom plate.

The importance of core damping is illustrated in Figs. 9 and 10. As can be seen from these figures, increasing damping in the core can reduce structural modal peaks. Such an effect is tiost beneficial in suppressing resonance of the fundamental mode because, as just stated, decreasing the core stiffness or top plate thickness doès not have much effect on the fundamental mode.

The effects of nonlinear stiffness and nonlinear damping of the core are shown in Fig. 11. Results shown in Fig. 11 indicate that a positive nonlineàrity might have a positive effect on response reduction above frequencies

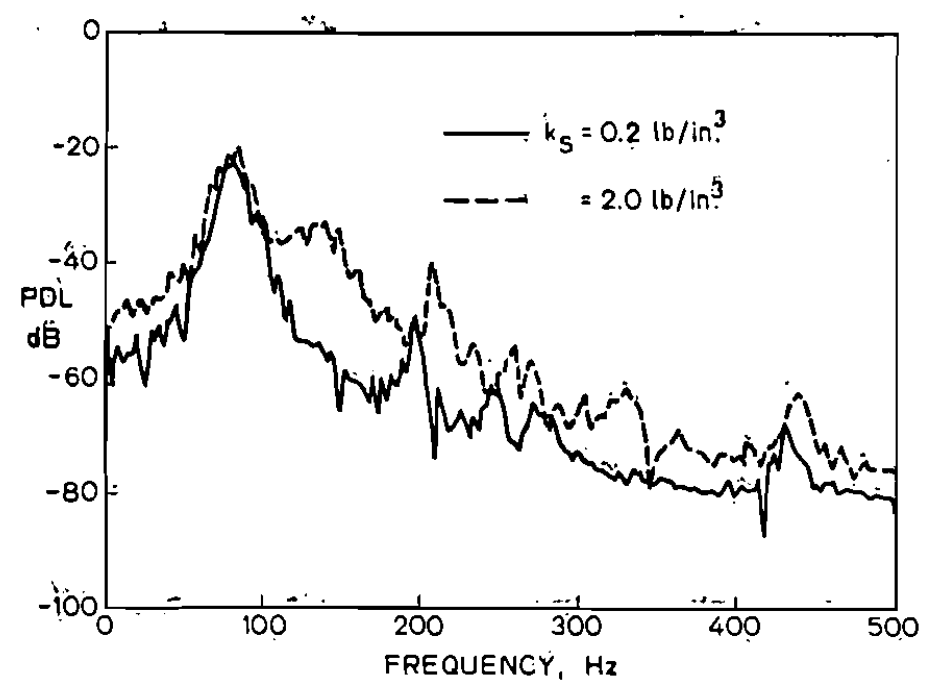

Fig. 7 Bottom plâte response for twio values of çare stiffnesses (Morite Carlo meiethod). 


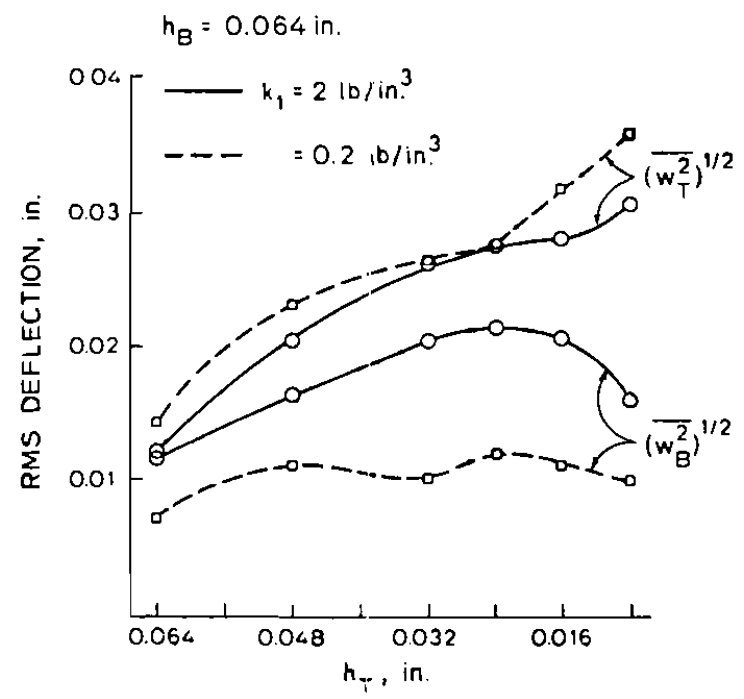

Fig. 8 Root-mean-square deflection resporise for different top plate thicknesses and two values of core stiffness.

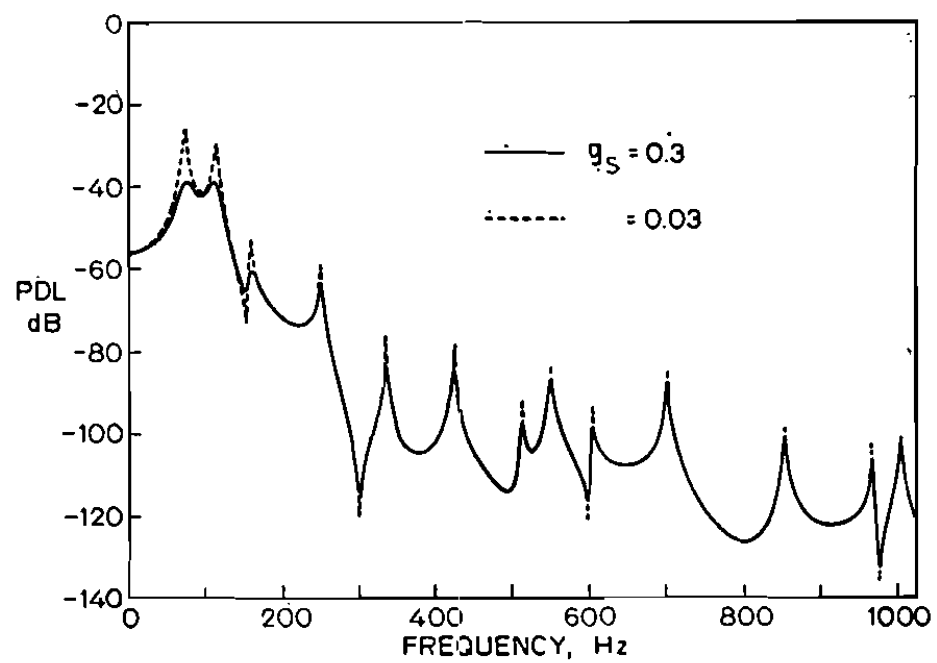

Fig. 9 Effect of core daimping on plate resjonse. 


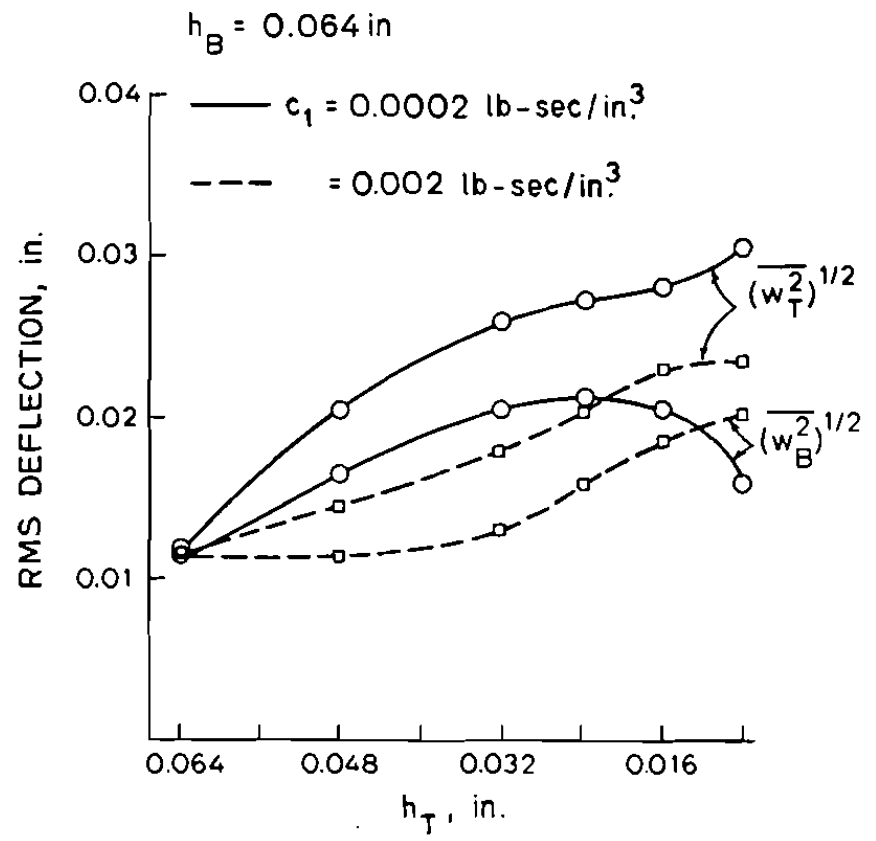

Fig. 10 Root-mean-square deflection response for different top plate thicknesses and two valucs of core damping.

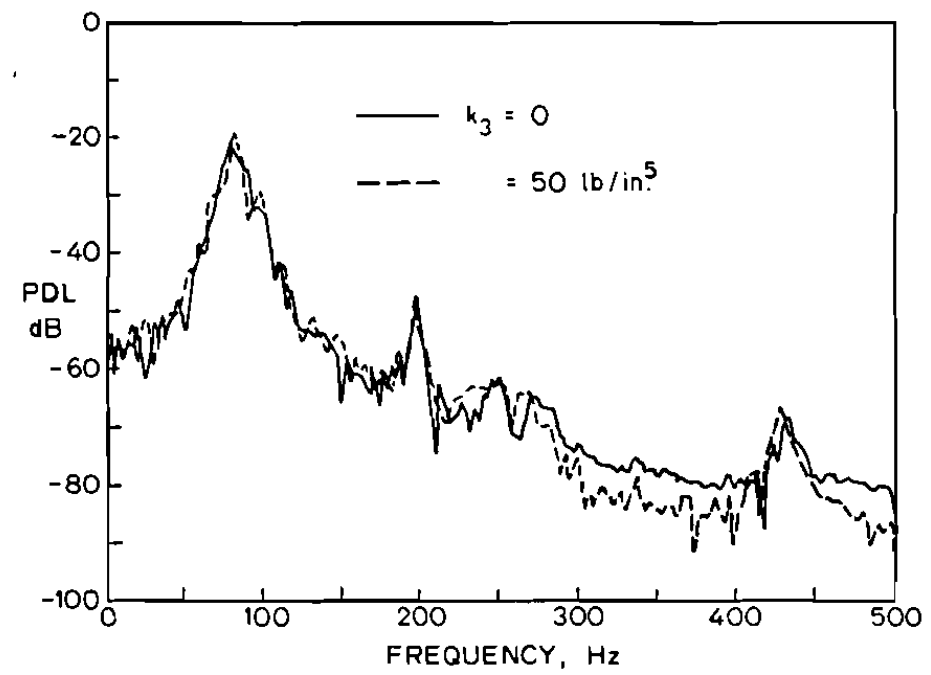

Fig. 11 Effect on nonlinear core stiffness on bottom plate response. 


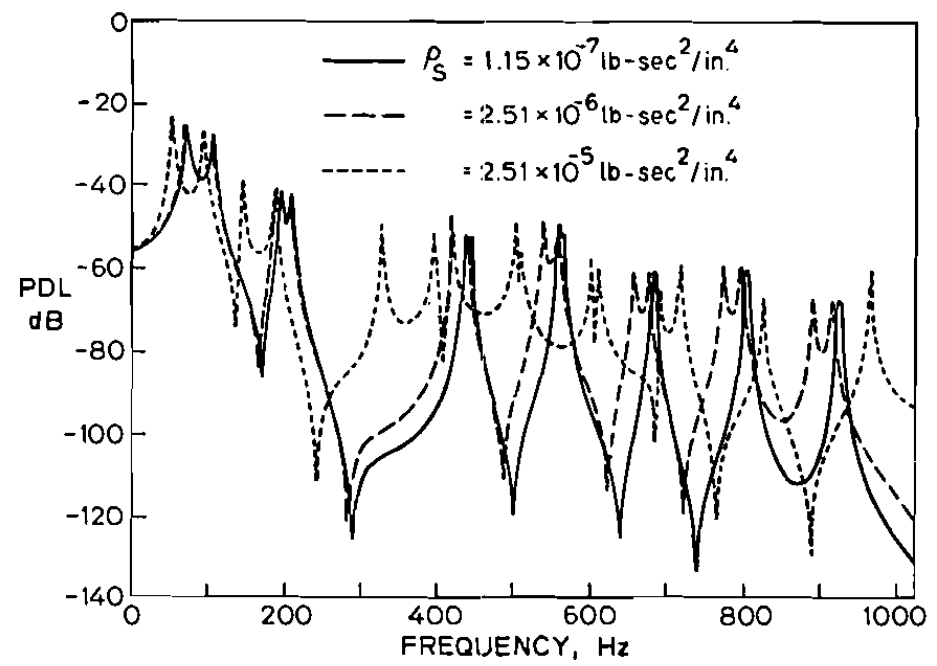

Fig. 12 Response of bottom plate for several values of core material density (PSD method).

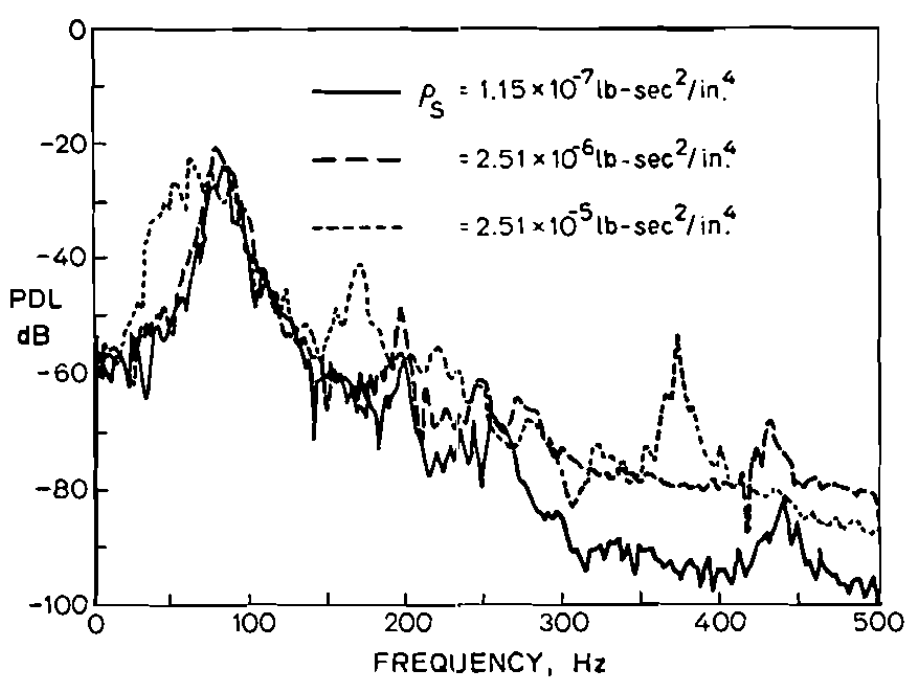

Fig. 13 Response of bottom plate for several values of core material density (Monte Carlo method). 
of $250 \mathrm{~Hz}$, while in the low frequency range (below $200 \mathrm{~Hz}$ ) no definite trend is detected. This could be attributed to the hardening effect of the core stiffness on suppressing dilatational modes. The nonlinear damping has very little influence on the response reduction, throughout the frequency range. Damping in the present system seems to be dominated by the linear damping term.

Mass of the core is apportioned to the top and bottom plates, as shown in Eqs. 1 and 3. Figure 12 depicts the response spectral density of the bottom plate for several values of core material density. In this case, thicknesses of the top and bottom plates are identical; i.e., $h_{T}=h_{B}=0.064$ in. Similar results are presented in Fig. 13 for $h_{B}=0.064 \mathrm{in}$. and $h_{T}=0.016 \mathrm{in}$., based on a time domain nonlinear analysis. With increasing core density, the flexural and dilatational modes tend to separate more and the resonant response increases and shifts toward lower frequencies. Thus, a double wall construction with a heavy core might not be beneficial to decrease the response of the bottom plate.

\section{v. CONCLUSIONS}

An analytical model has been developed to predict the nonlinear response of a double wall sandwich panel system that is subjected to random loading. Results indicate that the response is strongly dependent on geometric and material properties of the double wall sandwich construction. Deflection response of the bottom plate can be controlled by proper selection of the core stiffness and top plate thickness. For a soft core and nonlinear response of the top plate, no distinct resonant peaks were observed at frequencies above the fundamental resonant frequency of the coupled system. By increasing damping in the core, the response peak at the fundamental frequency can also be suppressed. This suggests that the viscoelastic damping factor of the core should be large in the vicinity of the fundamental natural frequency of the coupled system.

A better response reduction of the bottom plate is achieved for a core with a light mass than for a core with a heavy mass. Such an observation is contradictory to that of single panel construction, where more added weight is considered to be beneficial to response and noise attenuation. However, for double wall construction, inertia coupling between the top and bottom plates is introduced through the core. A heavier core tends to induce stronger coupling and larger response of the bottom plate. The effect of nonlinear stiffness and nonlinear damping of the soft core on response reduction is generally favorable, but the contributions are not very large. 


\section{APPENDIX}

The terms used are

$$
\begin{gathered}
Z_{i j k l m n r s}=(n r-m s)\left[F_{i j k l m n r s}(m+r, n+s)+\bar{F}_{i j k l m n r s}(m-r, n-s)\right] \\
+(n r+m s)\left[F_{i j k l m n r s}(m-r, n+s)+F_{i j k l m n r s}(m+r, n-s)\right] \\
\beta(p, q)=\left\{\begin{aligned}
1, & p=q \neq 0 \\
-1, & p=-q \neq 0 \\
0, & \text { otherwise }
\end{aligned}\right.
\end{gathered}
$$

where

$$
\alpha(p, q)= \begin{cases}1 /[(p+q) \pi]+1 /[(p+q) \pi], & (p+q) \text { and }(q-p) \text { odd } \\ 0, & (p+q) \text { and }(q-p) \text { even } \\ 1 /[(p+q) \pi], & (p+q) \text { odd and }(q-p) \text { even } \\ 1 /[(q-p) \pi], & (p+q) \text { even and }(q-p) \text { odd }\end{cases}
$$

$$
\begin{gathered}
\gamma(p, q)= \begin{cases}2, & p=q=0 \\
1, & p=q \neq 0 \\
0, & \text { otherwise }\end{cases} \\
F_{i j k \text { lmnrs }}(G, H)=\{2 G H k l[\beta(i+k, G)+\beta(i-k, G)][\beta(j+l, H) \\
+\beta(j-l, H)-\left(H^{2} k^{2}+G^{2} l^{2}\right)[\gamma(i+k, G) \\
-\gamma(i-k, G)] \cdot[\gamma(j+l, H)-\gamma(j-l, H)]\} \\
/\left[G^{2}+H^{2}(a / b)^{2}\right]^{2} \\
\bar{F}_{i j k l m n r s}(G, H)= \begin{cases}0, & G=H=0 \\
F_{i j k l m n r s}(G, H), & \text { otherwise }\end{cases}
\end{gathered}
$$

\section{ACKNOWLEDGMENT}

This work was supported by NSF Grant MEA 80-18339. 


\section{REFERENCES}

1. C. Mei, "Large Amplitude Response of Complex Structures Due to High Intensity Noise," Technical Report AFFDL-TR-79-3028, Wright-Patterson Air Force Base, Ohio, April 1979.

2. L. D. Jacobs and D. R. Lagerquist, "Finite Element Analysis of Complex Panel to Random Loads," Technical Report AFFDL-TR-68-44, Wright-Patterson Air Force Base, Ohio, 1968, pp. 55-59.

3. R. Vaicaitis, "Noise Transmission by Viscoelastic Sandwich Panels," NASA TN D-8516, August 1977.

4. C. L. Dym and M. A. Lang, Transmission of sound through sandwich panels, $J$. Acoust. Soc. Am. 56(5): 1523-1532 (November 1974).

5. C. P. Smolenski and E. M. Krokosky, Dilatational-mode sound transmission in sandwich panels, J. Acoust. Soc. Am. 54(6): 1449-1457 (December 1973).

6. A. M. Freudenthal and M. P. Bieniek, "Forced Vibrations of Sandwich Structures," WADD Technical Report 60-307, U.S. Air Force, January 1961.

7. R. D. Ford, P. Lord, and A. W. Walker, Sound transmission through sandwich constructions, J. Sound Vib. 5(1): 9-21 (January 1967).

8. S. Timoshenko and S. Woinowsky-Krieger, Theory of Plates and Shells, 2nd ed., McGraw-Hill, New York, 1959.

9. C.-Y. Chia, Nonlinear Analysis of Plates, McGraw-Hill, New York, 1980.

10. T. Caughey, Nonlinear theory of random vibrations, in Advances in Applied Mechanics, Vol. II (C. S. Yih, ed.), Academic, New York, 1971.

11. W. D. Iwan, Application of nonlinear analysis techniques, in Applied Mechanics in Earthquake Engineering, Vol. 8 (W. D. Iwan, ed.), ASME AMD, 1974, pp. 135-161.

12. S. H. Crandall, Nonlinear problems in random vibrations, in Seventh International Conference on Nonlinear Vibration, Vol. I1, Berlin, 1977, pp. 215-244.

13. T. K. Caughey, Derivation and application of the Fokker-Planck equation to discrete nonlinear dynamic systems subjected to white random excitation, J. Acoust. Soc. Am. 35: 1683-1692 (November 1973).

14. S. H. Crandall, Perturbation techniques for random vibration of nonlinear systems, J. Acoust. Soc. Am. 35: 1700-1705 (November 1963).

15. G. V. Anand and K. Richard, Nonlinear response of a string to random excitation, Int. J. Non-Linear Mech. 9: 251-260 (1974).

16. W. D. Iwan and I. M. Yang, Application of statistical linearization technique to nonlinear multidegree-of-freedom systems, J. Appl. Mech. 39: 545-550 (June 1972).

17. P.-T. D. Spanos, Stochastic linearization in structural dynamics, Appl. Mech. Rev. 34(1): 1-8 (1981).

18. M. Shinozuka and C.-M. Jan, Digital simulation of random processes and its applications, J. Sound Vib. 25(1): 111-128 (1972).

19. J.-N. Yang, Simulation of random envelop processes, J. Sound Vib. 21(1): 73-85 (1972).

20. M. Shinozuka, Monte Carlo solution of structural dynamics, Int. J. Comput. Struct. 2: 855-874 (1972).

21. R. Vaicaitis, C.-M. Jan, and M. Shinozuka, Nonlinear panel response from a turbulent boundary layer, AIAA J. 10(7): 895-899 (1972). 
22. J.-N. Yang, On the normality and accuracy of simulated random processes, $J$. Sound Vib. 26(3): 417-428 (1973).

23. M. Shinezuka, Digital simulation of random processes in engineering mechanics with the aid of FFT technique, in Stochastic Problems in Mechanics (S. T. Ariaratnam and H. H. E. Leipholz, eds.), University of Waterloo Press, 1974.

24. R. Vaicaitis, M. Shinczuka and M. Takeno, Response analysis of tall buildings to wind loading, J. Struct. Div., ASCE 101(ST3): 585-600 (March 1975).

25. V. V. Bolotin, Nonconservative Problents of the Theory of Elastic Stability, Pergamon, Oxford, 1963.

26. R. Vaicaitis, Nonlinear pancl response to nonstationary wind forces, J. Eng. Mech. Div., ASCE 101(EM4): 333-347 (August 1975).

27. E. H. Dowell, Transmission of noise from a turbulent boundary layer through a flexible plate into a closed cavity, J. Acoust. Soc. Am. 46(1, Part 2): 238-252 (July 1969).

28. P. W. Smith Jr., Response of non-linear structures to random excitation, J. Acoust. Soc. Am. 34(6): 827-835 (1962).

29. R. Vaicaitis and H.-K. Hong, "Noise Transmission through Nonlincar Sandwich Panels," AIAA 8th Aeroacoustic Conference, Atlanta, Georgia, April 1983, Paper No. 83-0696.

Received November 1983 